\title{
Desarrollo de la Teoría de la Mente entre los 5 y 6 años
}

\section{Development of Theory of Mind at five and six years old}

\author{
Rivas-García, S.M.; Hidalgo-Ruzzante, N.; Gutiérrez-García, G.; Bergés, G.D.; Caracuel, A.
}

Centro de Investigación Mente, Cerebro y Comportamiento-CIMCYC.

Universidad de Granada.

Correspondencia: Sandra María Rivas-García. smrivas@ugr.es

Resumen: La teoría de la mente (ToM) es la capacidad para reconocer los estados mentales de otras personas y de uno mismo para así poder actuar en consecuencia. Los objetivos fueron determinar si la adquisición de los ítems que sirven para evaluar la ToM entre los 5 y 6 años sigue una estructura jerárquica y cuáles son sus relaciones con otros factores. Método: 73 niños con una media de edad de 6.2 años fueron evaluados con una escala de la ToM de 7 ítems adaptados al español y una subescala de lenguaje pragmático. El análisis de Rasch mostró que la adquisición de los ítems de la escala de la ToM sigue un orden jerárquico, ligeramente distinto al de otras culturas. Se encontraron asociaciones significativas de ítems de la ToM con lenguaje pragmático y convivencia con hermanos/as. El desarrollo de los diferentes hitos que componen la Teoría de la Mente entre los 5 y 6 años se produce de forma jerárquica, con un orden que varía entre diferentes culturas. La adquisición de varios hitos de la ToM a estas edades se asocia con el grado de desarrollo de la capacidad linguística pragmática de los niños/as y su convivencia con hermanos/as, especialmente si son hermanos/as mayores.

Palabras clave: Teoría de la mente, Cognición social, Niños, Cultura, Lenguaje pragmático, Hermanos.
Abstract: Theory of Mind (ToM) is the ability to recognize the mental states of others and oneself in order to act accordingly. Objectives were to determine if the acquisition of the items to evaluate ToM in children of 5 and 6 years old follows a hierarchical structure, and relationships with other factors. Method: 73 children with a mean age of 6.2 years were evaluated with a ToM scale of 7 items adapted to Spanish, and a subscale of pragmatic language. Rasch analysis showed that the acquisition of the items of the ToM scale follows a hierarchical order, slightly different from other cultures. Significant associations between items of the ToM and pragmatic language and coexistence with siblings were found. Development of the different milestones that make up the ToM of children of 5 and 6 years old takes place in a hierarchical way, with an order that varies between different cultures. The acquisition of several ToM milestones at these ages is associated with the degree of development of children's pragmatic linguistic ability and their coexistence with siblings, especially if they are older siblings.

Keywords: Theory of mind, Social cognition, Children, Culture, Pragmatic language, Siblings.

\section{Introducción}

El sistema social del ser humano hace necesario que desarrollemos una capacidad altamente adaptativa que nos permita hacernos una idea de cómo se sienten los demás, qué intenciones tienen y anticipar cuál va a ser su comportamiento. Denominada Teoría de la Mente (ToM), esta habilidad implica la comprensión de los estados mentales de los demás (creencias, emociones, intenciones e intereses) y la predicción de su comportamiento, así como comprender que esos estados pueden ser diferentes de los de uno mismo y también diferir de la realidad [1].

La ToM está conformada por una serie de competencias de distintos niveles de complejidad entre las que destacan: 
Reconocimiento facial de emociones. En su desarrollo y funcionamiento está implicada la amígdala, encargada de detectar las expresiones de emociones básicas. El desarrollo deficitario de esta capacidad básica dificultará el reconocimiento de las emociones experimentadas por las otras personas y pondrá en compromiso el funcionamiento adecuado de la ToM [2].

Creencias de primer y segundo orden. Las denominadas creencias son la información necesaria para que la persona resuelva aquellas situaciones problemáticas en las que es preciso tener en cuenta el conocimiento previo del que disponen los otros individuos. La diferencia entre creencias de primer y segundo orden radica, fundamentalmente, en el grado de complejidad de las situaciones planteadas, por lo que se adquieren de forma gradual. Para evaluar el desarrollo adquirido en la comprensión de las creencias de los demás, se plantean una serie de ítems cuya resolución exige que se tengan en cuenta los pensamientos o conocimiento sobre la situación que tienen las otras personas. Uno de los pilares de la ToM es la comprensión de que las personas pueden estar equivocadas debido a que se han producido circunstancias novedosas de las que ellos no han sido conscientes [3]. Los ítems sobre las creencias de primer orden, también denominadas falsas creencias, son resueltos satisfactoriamente por la mayoría de los niños/as a la edad de 5 años [4].

- Comunicaciones metafóricas e historias extrañas, que incluyen la ironía, la mentira y la mentira piadosa. Son aspectos muy relevantes en la comprensión de la comunicación social, para cuya evaluación se han diseñado relatos en los que el mensaje principal se encuentra enmascarado gracias al uso de la ironía y la mentira piadosa [5].

Meteduras de pata. La sensibilidad social se evalúa mediante el planteamiento de historias en las que la persona debe ponerse en lugar de los protagonistas. Baron-Cohen, O'Riordan, Stone, Jones \& Plaisted [6] encontraron que estas historias implicaban una gran dificultad para los niños con síndrome de Asperger.

Expresión emocional a través de la mirada. La capacidad de conocer los sentimientos de otras personas observando su mirada es determinante para la ToM y existen multitud de test consistentes en presentar imágenes de los ojos. En algunos casos se evalúan aspectos emocionales complejos, por lo que para facilitar el reconocimiento se presentan distintas alternativas de respuesta [7].

Algunos autores han estudiado la relación entre la adquisición de la ToM y factores como el lenguaje, las relaciones con iguales, el nivel socioeconómico o determinadas patologías. En cuanto al lenguaje, la adquisición de la complejidad de los componentes más sofisticados de la ToM parece requerir del progreso en las competencias lingüísticas. A medida que aumenta el léxico y la complejidad sintáctica, el desarrollo de la ToM es más rápido y eficiente debido a que se comprenden de forma más adecuada las acciones tanto ajenas como propias [8]. La importancia del lenguaje para el desarrollo óptimo de la ToM se aprecia en un estudio en el que un grupo de niños/as sordos de padres oyentes presentaba un menor nivel en la ToM que los niños/as sordos de padres también sordos. Los autores atribuyen que en el segundo caso el desarrollo del lenguaje es mayor debido a un mejor nivel de comunicación entre hijos y padres que habían aprendido el lenguaje de signos desde la infancia [9]. Hughes et al., [10] también pusieron de manifiesto la relación del lenguaje con el desarrollo de la ToM en un estudio con niños/as de 5 años donde las diferencias individuales en la comprensión de las creencias falsas se asociaban a la habilidad verbal de los niños, que dependía de la variabilidad de los entornos linguiísticos, fundamentalmente del tipo de conversación familiar. Shahaeian [11] encontró asociación entre el nivel alcanzado en la ToM y la interacción con otros niños/as de edad similar. Los niños/as con hermanos/as de edad parecida potenciaron su ToM en función del tiempo que pasaban interaccionando con ellos en el hogar. La presencia de hermanos/as parece influir por mediación de la cantidad de interacción con ellos. Los resultados han mostrado que la influencia se puede ejercer tanto por hermanos/as mayores [12] como menores [13]. Respecto al nivel socioeconómico 
familiar, Shahaeian [11] comparó el nivel de desarrollo de la ToM en niños/as de diferentes clases sociales y económicas y no encontró ninguna asociación con el desarrollo de esta capacidad.

En cuanto a patologías del desarrollo en las que la ToM puede estar comprometida, destacan el autismo y el síndrome de Asperger [14] dónde los déficits en la adquisición de la ToM se asocian a efectos negativos en la evolución psicológica y cognitiva del individuo. Cómo ejemplos encontramos que son frecuentes los déficits en el reconocimiento de las falsas creencias de los demás, que se han asociado a las dificultades de socialización que presenta esta población [14]. También pueden tener dificultades para deducir, en base a su comportamiento, qué están pensando los demás y para incorporar lo que les intentan transmitir otros mediante advertencias o consejos [15]. En muchos de estos casos también se presentan retrasos en las competencias lingüísticas [8] que apoyan la relación entre lenguaje y ToM.

La influencia del entorno cultural es otro campo de interés en la investigación del desarrollo de la ToM. Estudios transculturales [10,16] revelan que la diferencia en las experiencias sociales influye en el desarrollo de la ToM. Estudios comparativos con niños/as de culturas orientales y occidentales [17] indican que el proceso evolutivo u orden de adquisición de los diferentes hitos que componen la ToM es parecido, pero no idéntico. Wellman \& Liu [18] utilizaron una escala formada por una recopilación de ítems clásicos con el objetivo de determinar estas diferencias. Cada ítem representa un hito evolutivo en la adquisición de la ToM en la primera infancia. Los elementos incluidos en la escala abarcaron desde las habilidades teóricamente más básicas (comprensión de que las personas pueden tener deseos diferentes) hasta las que a priori se plantearon como más sofisticadas (entender las emociones fingidas). Los resultados de 140 niños/as de Beijing (China) y 135 niños/as de Estados Unidos y Australia mostraron la existencia una ligera diferencia en el orden de adquisición que afectaba a dos de los hitos y que fueron atribuidas por los autores al efecto de la cultura [17].

\section{Objetivo}

En el presente estudio se ha realizado una adaptación española de los ítems utilizados en dos estudios del equipo de Wellman [17,18] para evaluar la Teoría de la Mente en niños/as. Las hipótesis del estudio fueron: 1) los hitos de la ToM tienen una estructura jerárquica, por lo que la adquisición de un hito o nivel determinado implica que se han adquirido los hitos de niveles inferiores y, 2) el grado de desarrollo de la ToM durante la primera infancia está relacionado de forma directamente proporcional con la edad, el nivel de lenguaje pragmático del niño/a y la convivencia con hermanos/as.

Los objetivos del estudio fueron: 1) determinar si la adquisición de los ítems utilizados por el equipo de Wellman para evaluar la Teoría de la Mente adaptados para niños/as españoles con edades comprendidas entre los 5 y 6 años sigue una estructura jerárquica, y 2) estudiar la relación entre el grado de desarrollo alcanzado en la Teoría de la Mente en la muestra de niños/as españoles y diversos factores: edad, nivel de lenguaje pragmático y convivencia con hermanos/as.

\section{Método}

\subsection{Participantes}

La muestra estuvo compuesta por 73 alumnos/as (56\% niñas) con una media de edad de 6,2 años $(\mathrm{DE}=0.58)$ de $3 e r$ curso de Educación Infantil y de 1er curso de Educación Primaria (60,3\% de la muestra) de un colegio del área metropolitana de Granada (España). El 78,1\% de los participantes tenía hermanos y el 42,5\% tenía hermanos mayores que ellos. El nivel socioeconómico promedio de las familias del centro era medio-alto. Los criterios de inclusión fueron tener una edad dentro del rango $\geq 5$ años $y \leq 7$, no tener 
diagnóstico del espectro del trastorno autista y que los tutores legales firmasen el consentimiento informado.

\subsection{Instrumentos}

Escala de la ToM en la infancia. Es una adaptación realizada por los autores para población infantil española partiendo de los 7 ítems que fueron utilizados en dos estudios realizados por el equipo de Wellman [9,18]. El objetivo de la escala fue medir el grado de desarrollo de la ToM de los participantes en el estudio. A continuación se enumeran el título, objetivo y orden de administración de los ítems adaptados:

1. Deseos Diversos. El objetivo es evaluar si el niño/a comprende que sus deseos no son comunes a todas las personas.

2. Diversas creencias. Objetivo: evaluar la comprensión que dos personas no tienen por qué tener la misma creencia sobre un objeto.

3. Acceso al conocimiento. Objetivo: evaluar el juicio sobre la ignorancia de otra persona a cerca del contenido de un recipiente.

4. Falsa creencia de contenido. Objetivo: evaluar si el niño/a es capaz de juzgar la falsa creencia de una persona sobre el contenido de un recipiente cuando él/ella conoce la verdad del contenido.

5. Falsa creencia explícita. Objetivo: evaluar la capacidad del niño/a para diferenciar entre su conocimiento y el de la otra persona.

6. Creencia-emoción. Objetivo: evaluar si el sujeto es capaz de comprender los sentimientos que otra persona puede experimentar en una situación determinada.

7. Emoción fingida. Objetivo: evaluar la capacidad de entender que una persona puede experimentar un sentimiento pero expresar otro diferente.

Cada ítem se puntúa en función de que la respuesta del niño/a indique que tiene adquirida la habilidad evaluada en cada ítem (puntuación=1) o no (puntuación=1), por lo que la puntuación global oscila entre 0 y 7.

Escena A de la Subescala de Lenguaje Pragmático de la Batería del Lenguaje Objetiva y CriterialScreening (BLOC-S-R) [19]. Compuesta de seis ítems que valoran la habilidad para utilizar el lenguaje en forma de saludos y despedidas, para hacer preguntas sobre el porqué y el cómo, para realizar requerimientos de acción, de atención y hacer comentarios de aprobación y desaprobación. Cada ítem se puntúa en función de que la respuesta del niño/a indique que tiene adquirida la habilidad evaluada en cada ítem (puntuación=1) o no (puntuación=1), por lo que la puntuación global oscila entre 0 y 6.

\subsection{Procedimiento}

Las evaluaciones con la escala de la ToM y de lenguaje pragmático se realizaron de forma individual, en una estancia conocida por los participantes, en horario lectivo. Las variables sociodemográficas de fecha de nacimiento, número de hermanos/as y cuáles eran hermanos/as mayores fueron respondidas por los padres en un cuestionario tras ser informados y aceptar su participación. El proyecto contó con el informe positivo del comité de ética de la Universidad de Granada.

\subsection{Análisis de datos}


Para el primer objetivo se realizó un análisis de Rasch mediante el software RUMM2020 para determinar si los ítems de la escala de la ToM se pueden ordenar de una forma jerárquica. El análisis de Rasch permite la distribución de ítems y de participantes utilizando un mismo tipo de escala logarítmica que tiene como punto de origen el 0 [20]. Para el segundo objetivo, mediante SPSS v24 se aplicó el coeficiente Rho de Spearman para determinar las asociaciones entre las puntuaciones de la escala de la ToM (global y de cada ítem) y el resto de variables: la edad, el número de hermanos y las puntuaciones de la subescala de lenguaje pragmático (global y de cada ítem). Finalmente se utilizaron tablas de contingencia para la comparación en cuanto a la adquisición de los diferentes ítems de la ToM separando la muestra en grupos de niños y de niñas, con y sin convivencia con hermanos y específicamente con y sin hermanos mayores.

\section{Resultados}

En la figura 1 se han resumido los porcentajes de superación de cada uno de los ítems de la escala de la ToM de la muestra completa y separando la muestra por curso escolar de infantil o primaria.

Figura 1. Niveles de desarrollo de la ToM por cursos. Se muestran los porcentajes de acierto en cada ítem de la ToM para los niños/as de Infantil y de Primaria, así como el promedio.

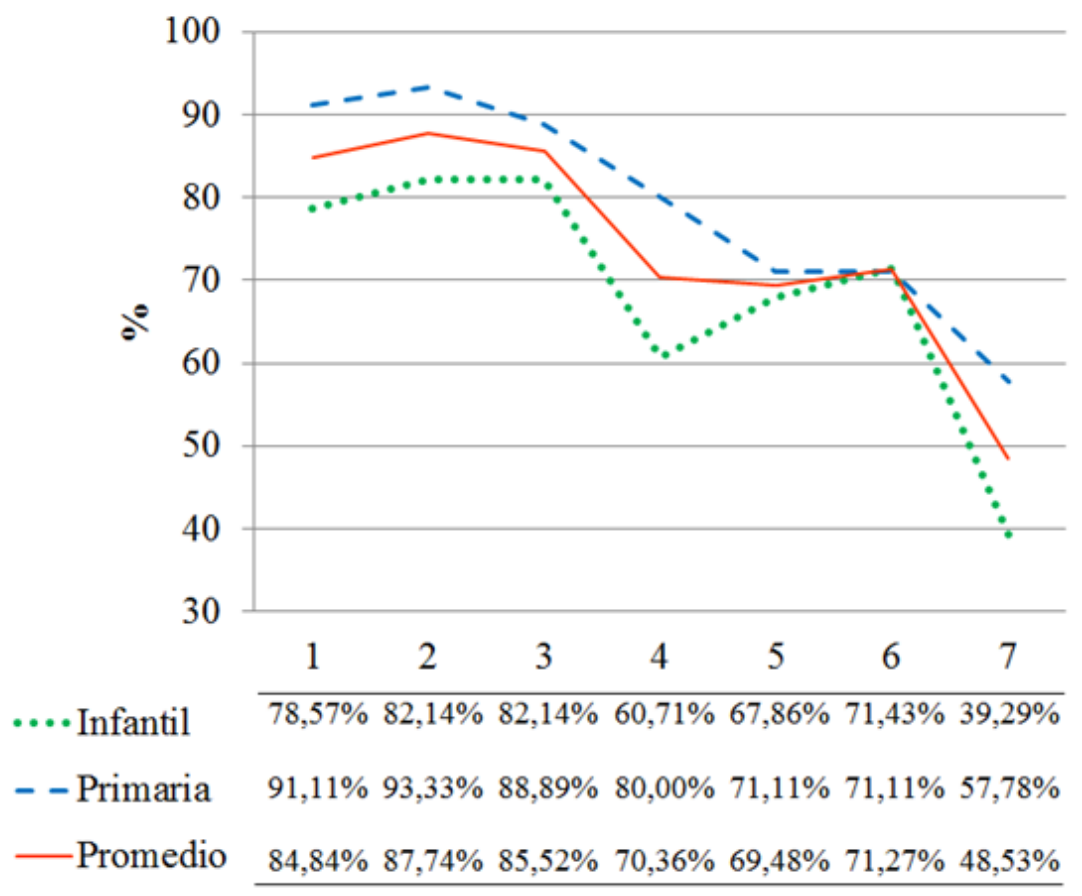

Niveles de Desarrollo ToM 
En la figura 2 se pueden ver los resultados para los ítems de la escala de lenguaje pragmático.

Figura 2. Niveles de desarrollo del lenguaje pragmático por cursos. Se muestran los porcentajes de acierto en cada ítem de la ToM para los niños/as de Infantil y de Primaria, así como el promedio.

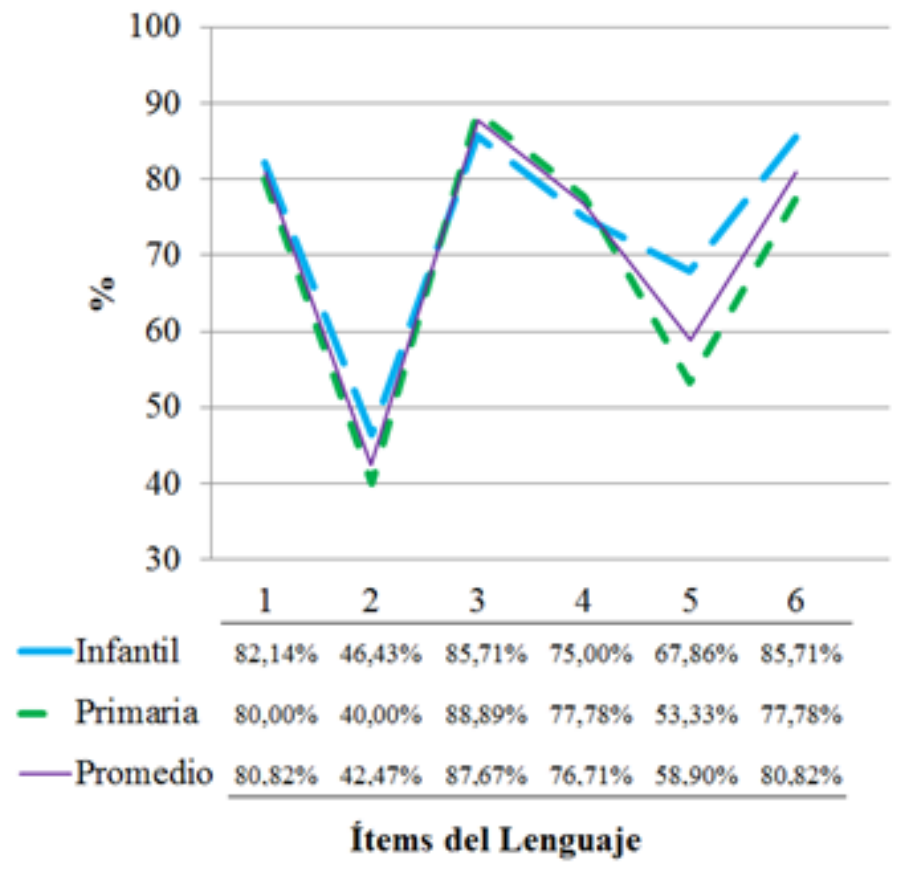

Para dar respuesta al primer objetivo y comprobar si el desarrollo de la ToM se produce de forma jerárquica, se realizó un análisis de Rasch con las puntuaciones de los participantes en cada uno de los 7 ítems de la escala de la ToM.

En la tabla 1 se muestra el orden de los niños/as en función de su grado de adquisición de los hitos de la ToM (columna 1). También se muestran los ítems dispuestos en función del nivel de dificultad para ser adquiridos (columnas 4 a 10). La jerarquía de los ítems, del hito más fácil de adquirir al más difícil, fue la siguiente: 2, 1, 3, 4, 6, 5 y 7. Se observa que los ítems 2 y 6 se situaron una posición anterior a la establecida a priori en base a estudios previos, por delante de los ítems 1 y 5 respectivamente. Los ítems 1 y 3 se han situado prácticamente igual en su localización en la escala logarítmica de grado de dificultad, e igual ocurre con los ítems 6 y 5.

A continuación, en la primera columna de la tabla 1, entre corchetes, aparece el orden de los niño/as de menor $(-1,724)$ a mayor $(2,767)$ grado de adquisición de la ToM y en la segunda fila, entre paréntesis, se indica la dificultad de los hitos, desde el ítem más fácil (-1.00) hasta el más difícil (1.76). 
Tabla 1. Escala Guttman.




Para el objetivo segundo de estudiar la relación entre el grado de desarrollo de la ToM y factores como la edad, el nivel de lenguaje pragmático y la convivencia con hermanos/as, se aplicó el coeficiente de correlación Rho de Spearman a las puntuaciones de la escala de la ToM (global y de cada ítem) y el resto de variables: la edad, el número de hermanos y las puntuaciones de la subescala de lenguaje pragmático (global y de cada ítem). En la tabla 2 se muestra una asociación entre la edad y el nivel de adquisición global de la ToM con tendencia a la significación ( $p=.093$ ). Sólo aparece una asociación estadísticamente significativa y de intensidad baja entre la edad y el hito 4 (Falsa creencia de contenido).

Tabla 2. Correlación entre los diferentes hitos del desarrollo de la ToM y la edad de los participantes (coeficiente Rho de Spearman).

\begin{tabular}{|c|c|c|c|c|c|c|c|c|}
\hline & & & & $\begin{array}{l}\text { Escala de } \\
\text { ToM }\end{array}$ & & & & \\
\hline & $\begin{array}{l}\text { Deseos } \\
\text { Diversos }\end{array}$ & $\begin{array}{l}\text { Creencias } \\
\text { Diversas }\end{array}$ & $\begin{array}{l}\text { Acceso al } \\
\text { Conocimiento }\end{array}$ & $\begin{array}{l}\text { Falsa } \\
\text { Creencia }\end{array}$ & $\begin{array}{l}\text { Falsa } \\
\text { Creencia } \\
\text { Explícita }\end{array}$ & $\begin{array}{l}\text { Creencia- } \\
\text { Emoción }\end{array}$ & $\begin{array}{l}\text { Emoción- } \\
\text { Fingida }\end{array}$ & $\begin{array}{l}\text { Puntuación } \\
\text { global } \\
\text { ToM }\end{array}$ \\
\hline Edad & 0.115 & 0.140 & 0.028 & $\begin{array}{l}0.275 \\
(p=.02)\end{array}$ & 0.080 & -0.032 & 0.195 & $\begin{array}{l}0.200 \\
(p=.09)\end{array}$ \\
\hline
\end{tabular}

En la tabla 3 se muestra una correlación con tendencia a la significación entre las puntuaciones globales de los ítems de la ToM y del lenguaje pragmático. Los resultados de las correlaciones ítem por ítem indican que tres de los siete hitos de la ToM (Acceso al conocimiento, Falsa creencia de contenido y Falsa creencia explícita) tienen asociaciones significativas con ítems del lenguaje pragmático (Saludos, Despedidas, Requerimientos de acción y comentarios de aprobación o desaprobación).

Tabla 3. Correlación entre los diferentes hitos del desarrollo de la ToM y el lenguaje (coeficiente Rho de Spearman).

Subescala de Lenguaje Pragmático

\begin{tabular}{|c|c|c|c|c|c|c|c|}
\hline Ítems de la ToM & Saludos & $\begin{array}{l}\text { Por qué } \\
\text { y cómo }\end{array}$ & Despedidas & $\begin{array}{l}\text { Requerimientos } \\
\text { directos de } \\
\text { acción }\end{array}$ & $\begin{array}{l}\text { Reclamaciones } \\
\text { de atención }\end{array}$ & $\begin{array}{l}\text { Comentarios } \\
\text { des/aprobación }\end{array}$ & $\begin{array}{l}\text { Puntuación } \\
\text { global } \\
\text { lenguaje }\end{array}$ \\
\hline Deseos Diversos & -0.084 & 0.106 & 0.213 & -0.021 & -0.084 & 0.020 & 0.082 \\
\hline $\begin{array}{l}\text { Creencias } \\
\text { Diversas }\end{array}$ & -0.051 & 0.129 & 0.134 & 0.024 & 0.070 & -0.166 & 0.102 \\
\hline $\begin{array}{l}\text { Acceso al } \\
\text { Conocimiento }\end{array}$ & $\begin{array}{c}0.229 \\
(p=.05)\end{array}$ & -0.056 & 0.091 & 0.075 & -0.002 & $\begin{array}{c}0.229 \\
(p=.05)\end{array}$ & 0.127 \\
\hline Falsa Creencia & 0.031 & 0.038 & $\begin{array}{c}0.234 \\
(p=.04)\end{array}$ & $\begin{array}{c}0.265 \\
(p=.02)\end{array}$ & -0.067 & 0.112 & $\begin{array}{c}0.199 \\
(p=.09)\end{array}$ \\
\hline $\begin{array}{l}\text { Falsa Creencia } \\
\text { Explicita }\end{array}$ & -0.076 & 0.090 & 0.114 & $\begin{array}{l}-0.282 \\
(p=.01)\end{array}$ & -0.053 & 0.081 & -0.045 \\
\hline Creencia- & 0.017 & 0.126 & 0.127 & 0.024 & -0.091 & 0.096 & 0.093 \\
\hline
\end{tabular}




\begin{tabular}{lccccccc} 
Emoción Fingida & -0.095 & 0.116 & 0.053 & 0.149 & -0.062 & -0.095 & 0.083 \\
$\begin{array}{l}\text { Puntuación global } \\
\text { ToM }\end{array}$ & -0.023 & 0.158 & $\begin{array}{c}0.222 \\
(p=.06)\end{array}$ & 0.083 & -0.048 & 0.102 & $\begin{array}{c}0.197 \\
(p=.09)\end{array}$ \\
\hline
\end{tabular}

En la tabla 4 se muestran correlaciones significativas entre el ítem 5 de la ToM (Falsa creencia explícita) y la convivencia con hermanos y entre el ítem 1 (Deseos diversos) y la convivencia con hermanos/as mayores.

Tabla 4. Correlación entre los diferentes hitos del desarrollo de la ToM y el número de hermanos (coeficiente Rho de Spearman).

\section{Escala de la ToM}

\begin{tabular}{|c|c|c|c|c|c|c|c|c|}
\hline & $\begin{array}{l}\text { Deseos } \\
\text { Diversos }\end{array}$ & $\begin{array}{l}\text { Creencias } \\
\text { Diversas }\end{array}$ & $\begin{array}{l}\text { Acceso } \\
\text { Conocimiento }\end{array}$ & $\begin{array}{l}\text { Falsa } \\
\text { Creencia }\end{array}$ & $\begin{array}{l}\text { Falsa } \\
\text { Creencia } \\
\text { explícita }\end{array}$ & $\begin{array}{l}\text { Creencia- } \\
\text { Emoción }\end{array}$ & $\begin{array}{l}\text { Emoción } \\
\text { Fingida }\end{array}$ & $\begin{array}{l}\text { Puntuación } \\
\text { global } \\
\text { ToM }\end{array}$ \\
\hline $\begin{array}{l}\text { Convivencia con } \\
\text { hermanos }\end{array}$ & -0.018 & 0.132 & -0.018 & 0.046 & $\begin{array}{l}0.229 \\
(p=.05)\end{array}$ & 0.175 & 0.073 & 0.175 \\
\hline $\begin{array}{l}\text { Convivencia con } \\
\text { hermanos mayores }\end{array}$ & $\begin{array}{l}0.262 \\
(p=.02)\end{array}$ & 0.124 & 0.020 & 0.031 & 0.081 & 0.117 & 0.016 & 0.121 \\
\hline
\end{tabular}

Finalmente se han agrupado los participantes para contrastar entre grupos algunos de los resultados anteriores aplicando análisis con tablas de contingencia y el estadístico $\chi^{2}$ de Pearson. Los resultados en la tabla 5 indicaron que el grupo que sí tenía hermanos mayores había obtenido una diferencia estadísticamente significativa en aciertos en el ítem sobre la comprensión de que los deseos de los demás pueden ser diferentes a los propios. En cuanto a las diferencias entre grupos por tener o no hermanos se ha encontrado una tendencia a la significación en el ítem sobre la falsa creencia explícita. En cuanto al sexo, tan solo se ha obtenido una tendencia a la significación en el ítem 3 (Acceso al conocimiento) que ha fue superado por el $92,7 \%$ de las niñas frente al $78,1 \%$ de los niños.

Tabla 5. Diferencias entre grupos (diferenciados por convivir con hermanos mayores, tener hermanos y el sexo) en las 7 pruebas de la batería de la Teoría de la Mente.

\begin{tabular}{|c|c|c|c|c|c|c|c|}
\hline \multicolumn{8}{|c|}{ Escala de la ToM } \\
\hline & $\begin{array}{l}\text { Deseos } \\
\text { Diversos } \\
\chi^{2}\end{array}$ & $\begin{array}{c}\text { Creencias } \\
\text { Diversas } \\
\chi^{2}\end{array}$ & $\begin{array}{c}\text { Acceso } \\
\text { Conocimiento } \\
\chi^{2}\end{array}$ & $\begin{array}{c}\text { Falsa } \\
\text { Creencia } \\
\chi^{2}\end{array}$ & $\begin{array}{c}\text { Falsa } \\
\text { Creencia } \\
\text { explícita } \\
\chi^{2}\end{array}$ & $\begin{array}{c}\text { Creencia- } \\
\text { Emoción } \\
\chi^{2}\end{array}$ & $\begin{array}{c}\text { Emoción } \\
\text { Fingida } \\
\chi^{2}\end{array}$ \\
\hline $\begin{array}{l}\text { Convivencia con } \\
\text { hermanos }\end{array}$ & 0.26 & 1.28 & 0.26 & 0.15 & $\begin{array}{c}3.84 \\
(p=.07)\end{array}$ & 2.25 & 0.39 \\
\hline $\begin{array}{l}\text { Convivencia con } \\
\text { hermanos } \\
\text { mayores }\end{array}$ & $\begin{array}{c}4.99 \\
(p=.04)\end{array}$ & 1.12 & 0.03 & 0.07 & 0.48 & 1.01 & 0.02 \\
\hline Sexo & 2.67 & 0.15 & $\begin{array}{c}3.22 \\
(p=.09)\end{array}$ & 1.40 & 1.49 & 0.40 & 1.10 \\
\hline
\end{tabular}

$\chi^{2}=$ Prueba Chi-cuadrado de Pearson 


\section{Discusión}

El primer objetivo del estudio fue determinar si la adquisición de los ítems utilizados por el equipo de Wellman para la evaluación de la Teoría de la Mente, adaptados para población española, seguían una estructura jerárquica en niños/as entre 5 y 6 años. Los resultados indican que los hitos evolutivos en el desarrollo de la ToM, representados por los ítems de la escala, se produce de forma jerárquica en niños/as españoles/as. Según el análisis de Rasch, la distribución de los ítems se ajusta a una matriz de Guttman que representa un orden de dificultad para ser adquiridos. Este orden es jerárquico y por tanto indica que haber adquirido un determinado hito implica una alta probabilidad de que los hitos situados en un orden de dificultad menor también hayan sido adquiridos. La secuencia de adquisición por los niños/as de la muestra de los ítems de la escala, ordenados desde el que se adquiere más fácilmente hasta el que resulta más difícil, y que por tanto son representativos de los hitos evolutivos de la ToM ha sido 2, 1, 3, 4, 6, 5 y 7 . Se han encontrado 2 cambios en el orden previsto de adquisición, que fue tomando de la secuencia del 1 al 7 obtenida por Wellman y Lui [18] en una muestra de niños/as americanos/as. En el presente estudio, los niños/as españoles han adquirido antes de lo esperado, el ítem número 2 (Creencias Diversas). La comprensión de que las personas tienen creencias diferentes a las suyas es el hito evolutivo de la ToM alcanzado en primer lugar por los niños/as españoles de la muestra. En cambio, este hito se encuentra en la segunda posición de dificultad para niños/as de culturas occidentales de habla inglesa [18] y en la tercera posición para los niños/as de China [17]. Los niños/as chinos adquieren antes el hito de la comprensión sobre el conocimiento o ignorancia de los otros que los americanos y australianos. Esta diferencia ha sido atribuida al énfasis en el conocimiento pragmático de la cultura china, que podría justificar que estos niños/as adquiriesen antes que los occidentales el juicio que permite diferenciar entre el conocimiento propio frente al de los demás [17].

El hito que alude a la comprensión de los deseos diversos se ha situado en segundo lugar en el orden de adquisición de la muestra española. Este hallazgo contradice lo que está establecido por el meta-análisis realizado por Wellman y Liu [18] y los datos con las muestras de niños chinos y occidentales [17]. Los estudios hasta el momento parecían indicar que, de forma universal, los niños/as logran juzgar de forma adecuada cuáles son los deseos de los demás antes que las creencias de los demás. No disponemos de datos con los que formular una hipótesis que explique adecuadamente esta diferencia entre la muestra de niños/as de cultura española y otras culturas. Al igual que en justificaciones previas sobre otros hitos de la ToM, es posible que sean aspectos educativos ligados a la cultura los que puedan explicar este hallazgo. Es posible que la cultura española fomente aún más que otras la necesidad de acceder al conocimiento que tienen los demás como forma de adaptación social. Sin embargo, esta explicación requiere un análisis transcultural profundo que podría ser objeto de futuras investigaciones en el campo de la cognición social. Por otro lado, debemos tener en cuenta que las diferencias en la escala de dificultad entre los hitos 2 y 1 son tan solo de 0,23 unidades en la escala de Rasch que aparece en la tabla 2 y que esta diferencia puede cambiar con muestras mayores. Respecto al ítem 6, que representa el hito de que la comprensión de que las emociones derivadas de las creencias pueden cambiar al mostrar la realidad que la creencia era errónea, ha presentado un grado de dificultad menor de lo esperado y se ha situado como el cuarto más fácil. Este hito ha resultado más fácil de adquirir en la muestra española que en la de niños/as americanos del estudio de Wellman y Lui [18]. Denham [21] también encontró que las creencias sobre las emociones se adquieren antes que algunos tipos de falsas creencias. Sin embargo, se trata de un hito escasamente estudiado, que no está incluido en ningún metaanálisis al que hayamos tenido acceso y que por tanto precisa de más investigación para llegar a establecer conclusiones claras. 
Varios hitos aparecen en la escala de dificultad del análisis de Rasch emparejados en grado de complejidad. El 1 (Deseos diversos) y el 3 (Acceso al conocimiento) tienen una dificultad similar de $-0,7$ y los ítems 6 (Creencia-emoción) y 5 (Falsa creencia explícita) están en torno a +0,3. La similitud en el orden entre los dos ítems de cada pareja indica que la superación del ítem de dificultad superior no implica una alta probabilidad de superación del otro ítem situado inmediatamente por debajo, ya que están casi paralelos en la secuencia. En cambio, la probabilidad de adquisición es mucho mayor sobre los hitos situados claramente por debajo en la escala de dificultad.

El segundo objetivo del estudio fue establecer la influencia en el desarrollo de la ToM de factores como la edad, la convivencia con hermanos/as y las dificultades de lenguaje. Respecto a la influencia de la edad en el desarrollo de la ToM, la falta de una asociación claramente significativa en la muestra del estudio se podría explicar por haber restringido la franja de edad a tan sólo 2 años (mínimo 5, máximo 7 años). Esta restricción justifica que la correlación significativa encontrada entre la edad y un hito de la ToM sea con el 4 (Falsa creencia de contenido) que se sitúa justo en el centro de la escala de Rasch sobre dificultad de los ítems y que por tanto es el que más variabilidad ha tenido en cuanto a respuestas acertadas y erróneas. Esta asociación entre la edad y el hito de la falsa creencia de contenido está en sintonía con los autores que postulan que la comprensión de que las creencias pueden ser falsas son un factor clave en el desarrollo de la ToM en la infancia [3].

Se han encontrado hallazgos sobre la asociación entre el desarrollo del lenguaje pragmático y la ToM. Aunque las puntuaciones globales en ambas variables no lleguen a ser significativas, a nivel de ítems concretos se aprecia una relación clara entre habilidades para la aplicación del lenguaje en contextos sociales y habilidades de la ToM, considerada el eje de la cognición social. Las respuestas adecuadas a los requerimientos directos de acción son una capacidad lingüística que se ha relacionado de una forma más directa con el nivel de desarrollo en la comprensión de las falsas creencias, tanto de contenido como explícitas. Parece lógico que en la adquisición de una adecuada comprensión de las creencias de los demás juegue un papel fundamental la capacidad lingüística necesaria tanto para hacer como para responder a los requerimientos de acción de los demás. La realización de y la respuesta a demandas de los demás podría facilitar el entendimiento de cuáles son los pensamientos e intenciones de otros sobre objetos y sobre las demás personas. Autores como Povinilli y Preus [3] avalan desde hace tiempo que la riqueza lingüística de los niños/as se corresponde con su desarrollo en la ToM. Actualmente, la literatura indica que el progreso en el lenguaje se ve reflejado en el desarrollo de la ToM [8]. Al igual que en otros estudios, el desarrollo de la ToM se ha asociado con tener hermanos/as, y de forma específica con que los hermanos sean de una edad mayor a la del niño/a que se encuentra en este proceso del desarrollo de la cognición social [13]. La asociación entre la adquisición de la comprensión de la existencia de deseos diversos por parte de otros y tener hermanos/as mayores resulta fácilmente comprensible. No es infrecuente que los deseos de los hermanos sean diferentes, y además es lógico que los de mayor edad sean más hábiles a la hora de imponer los suyos y propiciar que los hermanos/as menores tomen mayor conciencia sobre la diferencia de los deseos de otros respecto a los suyos.

No se han encontrado diferencias significativas en la adquisición de los hitos de la ToM entre niños y niñas. Este hallazgo es concordante con la literatura [12] sin embargo, la tendencia a la significación en uno de los ítems de la escala de la ToM indica que se trata de un aspecto que podría investigarse teniendo en cuenta habilidades específicas que componen la ToM y las pruebas que se utilizan para evaluar este tipo de diferencias asociadas al sexo en distintas etapas evolutivas. 
En conclusión, el desarrollo de los diferentes hitos que componen la Teoría de la Mente entre los 5 y 6 años se produce de forma jerárquica, con un orden que varía entre diferentes culturas. La adquisición de varios hitos de la ToM a estas edades se asocia con el grado de desarrollo de la capacidad lingüística pragmática de los niños/as y su convivencia con hermanos/as, especialmente si son hermanos/as mayores.

Agradecimientos. Estudio financiado con una ayuda para la Formación de Profesorado Universitario del Ministerio de Educación de España.

\section{Referencias}

1. Cassel A, McDonald S, Kelly M, Togher L. Learning from the minds of others: A review of social cognition treatments and their relevance to traumatic brain injury. Neuropsychol Rehabil. 30 de noviembre de 2016;0(0):1-34.

2. Brüne M. Emotion recognition, 'theory of mind,' and social behavior in schizophrenia. Psychiatry Res. 28 de febrero de 2005;133(2):135-47.

3. Povinelli DJ, Preuss TM. Theory of mind: evolutionary history of a cognitive specialization. Trends Neurosci. septiembre de 1995;18(9):418-24.

4. Baron-Cohen S, Leslie AM, Frith U. Does the autistic child have a "theory of mind" ? Cognition. octubre de 1985;21(1):37-46.

5. Happé FGE. An advanced test of theory of mind: Understanding of story characters' thoughts and feelings by able autistic, mentally handicapped, and normal children and adults. J Autism Dev Disord. 1 de abril de 1994;24(2):129-54.

6. Baron-Cohen S, O'Riordan M, Stone V, Jones R, Plaisted K. Recognition of Faux Pas by Normally Developing Children and Children with Asperger Syndrome or High-Functioning Autism. J Autism Dev Disord. 1 de octubre de 1999;29(5):407-18.

7. Baron-Cohen S, Wheelwright S, Hill J, Raste Y, Plumb I. The "Reading the Mind in the Eyes" Test Revised Version: A Study with Normal Adults, and Adults with Asperger Syndrome or Highfunctioning Autism. J Child Psychol Psychiatry. 1 de febrero de 2001;42(2):241-51.

8. Resches M, Serrat E, Rostan C, Esteban M. Language and Theory of Mind: A multidimensional approach. Infancia Aprendiz. 1 de enero de 2010;33(3):315-33.

9. Wellman HM, Fang F, Peterson CC. Sequential Progressions in a Theory-of-Mind Scale: Longitudinal Perspectives. Child Dev. 1 de mayo de 2011;82(3):780-92.

10. Hughes C, Jaffee SR, Happé F, Taylor A, Caspi A, Moffitt TE. Origins of Individual Differences in Theory of Mind: From Nature to Nurture? Child Dev. 1 de marzo de 2005;76(2):356-70. 
11. Shahaeian A. Sibling, Family, and Social Influences on Children's Theory of Mind Understanding: New Evidence From Diverse Intracultural Samples. J Cross-Cult Psychol. 1 de abril de 2015;46:116.

12. McAlister A, Peterson C. A longitudinal study of child siblings and theory of mind development. Cogn Dev. 1 de junio de 2007;22(2):258-70.

13. Prime H, Plamondon A, Pauker S, Perlman M, M. Jenkins J. Sibling cognitive sensitivity as a moderator of the relationship between sibship size and children's theory of mind: A longitudinal analysis. Cogn Dev. 1 de julio de 2016;39(Supplement C):93-102.

14. White S, Hill E, Happé F, Frith U. Revisiting the Strange Stories: Revealing Mentalizing Impairments in Autism. Child Dev. 1 de julio de 2009;80(4):1097-117.

15. Valdez D, editor. Autismo: enfoques actuales para padres y profesionales de la salud y la educación. Buenos Aires: Fundec; 2001.

16. Lecce S, Hughes C. «The Italian job?»: comparing theory of mind performance in British and Italian children. Br J Dev Psychol. noviembre de 2010;28(Pt 4):747-66.

17. Wellman HM, Fang F, Liu D, Zhu L, Liu G. Scaling of theory-of-mind understandings in Chinese children. Psychol Sci. diciembre de 2006;17(12):1075-81.

18. Wellman HM, Liu D. Scaling of Theory-of-Mind Tasks. Child Dev. 1 de marzo de 2004;75(2):52341.

19. Puyuelo Sanclemente M, editor. Bloc screening-R: evaluación del lenguaje. Nueva ed. revisada y ampliada. Barcelona: Elsevier Masson; 2007. 1 p.

20. Pallant JF, Tennant A. An introduction to the Rasch measurement model: an example using the Hospital Anxiety and Depression Scale (HADS). Br J Clin Psychol. 2007;46(Pt 1):1-18.

21. Denham SA. Social Cognition, Prosocial Behavior, and Emotion in Preschoolers: Contextual Validation. Child Dev. 1986;57(1):194-201.

Trabajo seleccionado por su calidad científica: X Congreso Nacional de Neuropsicología. Federación de Asociaciones de Neuropsicología Españolas (FANPSE). Valencia. España. 2018. 